\title{
Covalently Bound Plasticized PVC Membranes for Solid State Ion-Selective Devices
}

\author{
Peter D. van der Wal ${ }^{\star}$, Renata Zielinska-Paciorek ${ }^{\mathrm{a}}$, and Nicolaas F. de Rooij
}

\begin{abstract}
A method is described that makes it possible to covalently link plasticized PVC membranes to glass and other oxide surfaces. It involves the addition of an amine-group containing silane coupling agent and a heating step. Using this method, ion-sensitive field effect transistors (ISFETs) were modified with ion-selective plasticized PVC-membranes. The ion-selective properties of these devices were similar to those of devices prepared without linking the membrane to the surface, while the adhesion was dramatically improved.
\end{abstract}

Keywords: Ion-selective electrodes · ISFETs $\cdot$ Membrane adhesion · Plasticized PVC

\section{Introduction}

Since their introduction more than 30 years ago, liquid membrane ion-selective electrodes using plasticized PVC membranes have become well established and highly relevant analytical tools. With the development of thin and thick film technology, there is an increasing interest in the fabrication of ion-selective electrodes on solid-state substrates. Typical examples of microfabricated devices are membrane modified ionsensitive field effect transistors (ISFETs) and planar coated wires. The former are prepared with silicon thin film technology, and the latter can be made by thin or thick film technology on a variety of substrates including silicon, ceramics, and polymeric materials. The driving force for using microfabrication techniques to manufacture ion-selective electrodes are the presumed low cost price and the small size of the devices.
For macro-electrodes it is not really an issue which material should be used as a membrane matrix. Although various polymer matrices such as polyurethane, silicone rubber and polyacrylates have been tested, plasticized PVC membranes are still considered to be the best choice. However, when changing from macro-electrodes to microfabricated devices, several problems may become acute. These include the adhesion of the ion-selective membrane to the substrate, the leaching of electroactive components from the membrane, the deposition of the membrane and the interface processes between the membrane and the transducer. A vast number of publications have appeared in the literature that address the aforementioned problems.

Regarding the membrane deposition technology, it is often mentioned that the membrane materials should be photo-polymerizable or -crosslinkable in order to be compatible with IC technology. However, the development step after UV-exposure involves washing the wafer with an organic solvent. Through this intimate contact of the cured membrane with the organic solvent, any compound that is not covalently bound may be leached out, leading to a deterioration of the final sensor properties. When aiming at fabrication of multisensors, different ion-selective membranes have to be handled in sequence. Hence, the first deposited and cured membranes will come into contact with the organic solutions of monomers for the later prepared ion-selective membranes, which bears the risk of cross-contamination. In our earlier studies on sensors based on photosensitive polysiloxanes [1], it was found that leaching of membrane components is a serious problem resulting in an increase of noise levels, whereas cross-contamination was not observed with the tested configurations. In conclusion, it appears that photo-polymerizable membrane materials are not the best choice for the fabrication of solid-state ion-selective devices.

Plasticized PVC is a more versatile matrix for ion-selective sensors. By choosing the appropriate plasticizer, it is even possible to fine tune the selectivity of the devices. A major problem, however, is the adhesion of the membrane to the substrate surface. Generally, membrane adhesion can be improved either by mechanical fastening or chemical anchoring. Examples of the former are the suspended gate polyimide mesh ISFET through which the plasticized PVC membrane is cast [2] and devices using plasticized PVC cast in cavities of etched through-holes of a silicon wafer [3]. Mechanical fastening has the disadvantage that it complicates the fabrication process and increases the costs considerably. Chemical anchoring of the membrane has only been described for matrices other than PVC since binding reactions with standard plasticized PVC membranes do not proceed at room temperature. Earlier attempts using silanes to bind PVC to oxide surfaces [4-6] were restricted to modified PVC or copolymers of PVC, and the bonds formed were prone to hydrolysis. 
Special treatments to increase the adhesion between plasticized PVC and substrates like glass were developed for industrial applications (see [7] and the references found therein). For example cationic styryl and amine group containing silanes have been described to improve the adhesion between PVC plastisols and glass at $180{ }^{\circ} \mathrm{C}$. Based on this knowledge, we started investigations on the adhesion of PVC membranes to glass substrates [8]. It was found that silane coupling agents with amine groups are capable of increasing the adhesion considerably, but heating is indispensable for good results (see also below). Surface silanization of the glass substrates was shown to be insufficient; instead, the silane had to be added to the membrane mixture.

The chemical anchoring method described here is very simple and can be used without any special equipment. Further, it uses commonly available chemicals like $\gamma$-APS. This is in contrast to another procedure [9] that basically uses the same anchoring reactions, but requires the synthesis of PVC modified with alkoxy silane groups.

The method described in the present paper shows clear advantages to the method of Boden et al. [9] as no special equipment or chemicals are necessary. A patent application for the method described here has been granted [10].

\section{Experimental}

\section{Reagents}

The solvents cyclohexanone and tetrahydrofuran (selectophore) were purchased from Fluka. The coupling agent 3-aminopropyltriethoxysilane (APS) was from ABCR, Germany. The ionophores valinomycin, ETH 5234 (calcium ionophore IV), nonactin and tetradodecylammonium nitrate (TDAN) and the ionic additives potassium tetrakis(4-chlorophenyl) borate $\left(\mathrm{KB}(\Phi \mathrm{Cl})_{4}\right)$, potassium tetrakis (3,5 bis(trifluoromethyl) phenyl) borate $\left(\mathrm{KB}\left(\Phi\left(\mathrm{CF}_{3}\right)_{2}\right)_{4}\right)$ were of selectophore quality from Fluka.

Poly(vinyl chloride), high molecular weight, and plasticizer bis(2-ethylhexyl) sebacate (DOS), both selectophore reagents, were purchased from Fluka.

All electrolyte solutions were made with salts of p.a. quality (Merck) in deionized water.

\section{ISFETS}

The ISFETs were prepared at the IMT facilities. These n-channel devices embedded in a p-well have dimensions $1.2 \times 3.0 \mathrm{~mm}$. The $\mathrm{Al}_{2} \mathrm{O}_{3}$ gate oxide was deposited by a CVD process. The gate is meander shaped, covering an area of $300 \mu \mathrm{m}$ long and $120 \mu \mathrm{m}$ wide. Around the gate, an oval shaped SU-8 (photosensitive epoxy resin) ring was deposited using photolithography; the inner dimensions of the ring are 300 by $500 \mu \mathrm{m}$. The $100 \mu \mathrm{m}$ thick ring is used to define the area in which the adhesive layer and the ion-selective membrane can be solvent-cast. The ISFETs were mounted on a printed circuit board, wire-bonded, and encapsulated using epoxy resin.

Measurements were performed using a custom made ISFET amplifier in a constant drain current mode $\left(\mathrm{I}_{\mathrm{D}}=100 \mu \mathrm{A}\right)$ with a constant drain-source voltage $\left(\mathrm{V}_{\mathrm{DS}}=0.5 \mathrm{~V}\right)$. A double junction $\mathrm{Ag} / \mathrm{AgCl}$ reference electrode with $1 \mathrm{M}$ lithium acetate bridge electrolyte was used. For the calculation of activity coefficients, the Debye-Hückel theory was used. Emf values were correc-ted for the liquid junction potentials using the Henderson equation. Selectivity coefficients were determined by the fixed interference method at background levels of $0.1 \mathrm{M}$ of the interfering ions; chloride salts were used for the cation-selective devices, and potassium salts for nitrate-selective devices.

\section{Ion-selective Membranes}

The adhesive layer mixture was composed of $100 \mathrm{mg}$ PVC, $200 \mathrm{mg}$ DOS, and 3 $\mathrm{mg}$ of APS in $3 \mathrm{ml}$ of solvent. A droplet of the solution was dispensed inside the SU-8 ring using a Hamilton syringe fitted with a flexible fused silica needle. The devices were left for evaporation of the solvent overnight, and then heated for $2 \mathrm{~h}$ at $120^{\circ} \mathrm{C}$. After cooling down, the ISFETs were packaged as described above. The ion-selective membranes were deposited in the last step on the encapsulated devices.

The composition of the ion-selective membranes is given in Table 1. The valinomycin containing membrane $\mathbf{1}$ was dispensed as a cyclohexanone solution and the other membranes dissolved in tetrahydrofuran. The membrane solution was dispensed over the gate area, also spreading out on the epoxy encapsulation material. The devices were then left for evaporation of the solvent overnight. Before use, they were conditioned in $0.1 \mathrm{M}$ solutions of the primary ion for several hours.

Table 1. Composition of the ion-selective membranes in wt\%.

$\begin{array}{llllccr}\text { membrane } & \text { PVC } & \text { DOS } & \begin{array}{l}\text { neutral } \\ \text { ionophore }\end{array} & \mathrm{KB}(\Phi \mathrm{Cl})_{4} & \begin{array}{c}\text { ionic additive } \\ \mathrm{KB}\left(\Phi\left(\mathrm{CF}_{3}\right)_{2}\right)_{4}\end{array} & \text { TDAN } \\ \mathbf{1} & 32.8 & 64.6 & 2.1 \text { (valinomycin) } & 0.5 & & \\ \mathbf{2} & 33.0 & 65.4 & 1.0 \text { (nonactin) } & & 0.6 & \\ \mathbf{3} & 33.0 & 64.7 & 1.5 \text { (ETH 5234) } & & 0.8 & \\ \mathbf{4} & 32.0 & 64.0 & & & 4.0\end{array}$




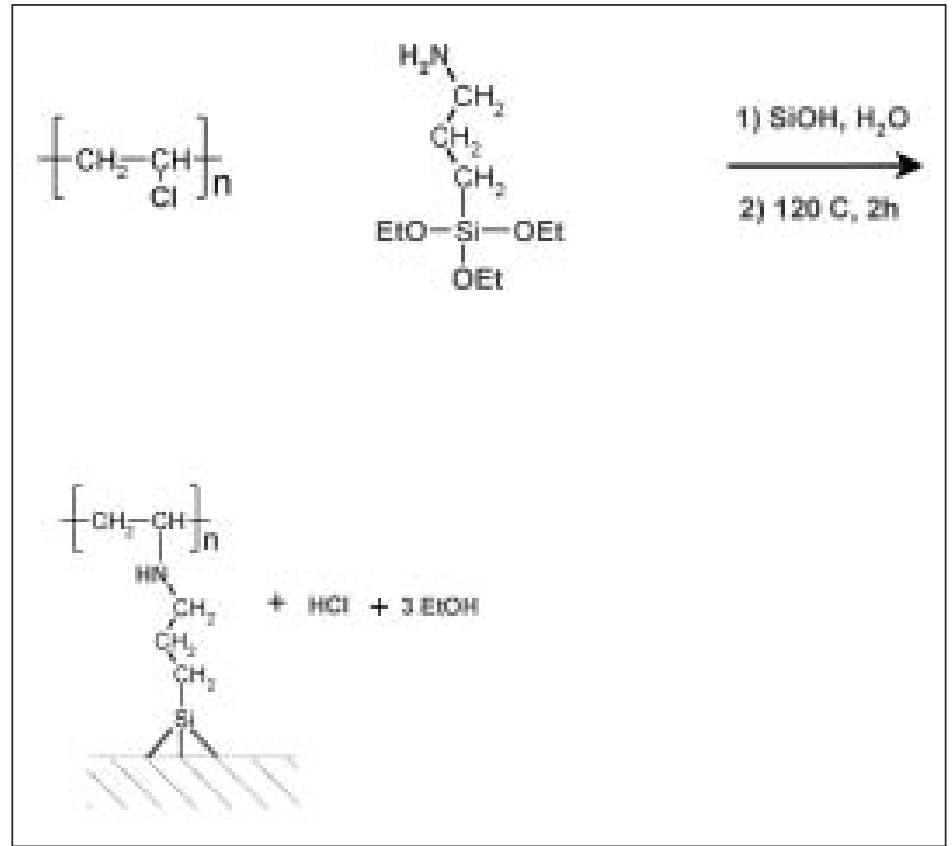

Fig. Schematic representation of the reactions between the silane coupling agent, the PVC-backbone and the oxide surface layer, ion-selective membranes were also cast on devices without the adhesive layer.

All the tested devices showed good ionselective properties with almost Nernstian slopes (see Table 2). Sensor characteristics such as linear ranges, detection limits and selectivities were similar for devices with and without adhesive layer.

The lifetime of these ion-selective sensors was at least in the order of two months. For devices with the adhesive layer the membrane adhesion was still excellent even after two months of continuous immersion. In contrast the membrane adhesion on the devices without adhesive layer turned out to be poor since the membranes could easily be removed, leaving no residue on the surface. Noteworthy is, however, that these latter devices may function correctly for several weeks if no mechanical or hydrodynamical force is applied to the membrane.

Further investigations are necessary regarding the long-term stability of these ion-selective sensors. Of special interest is the establishment of a thermodynamically well-defined interface between membrane and the oxide surface. For membranemodified ISFETs, it is possible to use an approach with an inner electrolyte that is buffered at an appropriate $\mathrm{pH}$. This can be realized by patterning a structure on the
Table 2. Slopes and selectivity coefficients of the membrane-modified ISFETs

\begin{tabular}{|c|c|c|c|c|c|c|c|c|}
\hline \multirow{2}{*}{$\begin{array}{c}\text { ISFET / } \\
\text { membrane }^{a}\end{array}$} & \multirow[t]{2}{*}{$\mathrm{i}$} & \multirow[t]{2}{*}{ slope } & \multirow[b]{2}{*}{$\mathrm{Na}^{+}$} & \multirow[b]{2}{*}{$\mathrm{K}^{+}$} & \multicolumn{3}{|c|}{$\log K_{i j} ; j=$} & \multirow[b]{2}{*}{$\mathrm{SO}_{4}^{2-}$} \\
\hline & & & & & $\mathrm{Mg}^{2+}$ & $\mathrm{Ca}^{2+}$ & $\mathrm{Cl}^{-}$ & \\
\hline $1 a$ & $\mathrm{~K}^{+}$ & $59.5 \pm 0.6$ & -3.6 & - & -4.3 & -4.6 & & \\
\hline $1 b$ & $\mathrm{~K}^{+}$ & $58.7 \pm 0.9$ & -3.6 & - & -4.3 & -4.6 & & \\
\hline $2 a$ & $\mathrm{NH}_{4}^{+}$ & $57.3 \pm 1.3$ & -3.0 & & -4.6 & -4.6 & & \\
\hline $2 b$ & $\mathrm{NH}_{4}^{+}$ & $58.1 \pm 1.1$ & -3.0 & & -4.6 & -4.6 & & \\
\hline $3 \mathbf{a}$ & $\mathrm{Ca}^{2+}$ & $27.3 \pm 1.3$ & -3.0 & -3.4 & -4.9 & - & & \\
\hline $3 b$ & $\mathrm{Ca}^{2+}$ & $28.7 \pm 0.7$ & -3.0 & -3.4 & -4.9 & - & & \\
\hline $4 a$ & $\mathrm{NO}^{3-}$ & $-57.9 \pm 2.0$ & & & & & -2.7 & -3.7 \\
\hline $4 b$ & $\mathrm{NO}^{3-}$ & $-59.0 \pm 0.5$ & & & & & -2.7 & -3.7 \\
\hline
\end{tabular}

a The membrane numbers correspond to the membrane compositions given in Table 1; a: without and $\mathbf{b}$ : with adhesive layer chip surface that consists of a double ring. In the inner ring, the inner electrolyte is dispensed either as a salt solution or a hydrogel. In the outer ring the adhesive layer is dispensed and after the curing step, the ionselective membrane is dispensed over the whole area. The inner electrolyte will take up water according to the average osmotic pressure acting on the sample solution side.

\section{Conclusions}

Plasticized PVC membranes can be covalently bound to oxide surfaces using a silane coupling agent with an amine group. In the proposed method, first an adhesive layer is bound to the surface, and then the ion-selective membrane is cast on top of this. The sensor characteristics like slope, linear range, detection limits, and selectivity were comparable for devices with and without adhesive layer. The lifetime of the sensors needs to be investigated in further detail, but is now no longer limited by the membrane adhesion; the latter is still excellent after two months of immersion. Further investigations on the signal stability of sensors prepared by the new method, are in full progress.

Received: July 14, 2003

[1] P.D. van der Wal, A. van den Berg, N.F. de Rooij, Sensors and Actuators B 1994, 18 19, 200.

[2] G. Blackburn, J. Janata, J. Electrochem. Soc.: Solid State Science and Technology 1982, 129, 2580.

[3] A. Uhlig, E. Lindner, C. Teutloff, U. Schnakenberg, R. Hintsche, Anal. Chem 1997, 69, 4032

[4] D.J. Harrison, L.L. Cunningham, X. Li, A. Teclemariam, D. Permann, J. Electrochem. Soc. 1988, 135, 2473.

[5] T. Satchwill, J.D. Harrison, J. Electroanal. Chem. 1986, 202, 75 .

[6] G.J. Moody, J.D.R. Thomas, J.M. Slater, Analyst 1988, 113, 1703.

[7] E.D. Plueddemann, 'Silane Coupling Agents', $2^{\text {nd }}$ Ed., Plenum Press, 1991

[8] P.D. van der Wal, N.F. de Rooij and M. Koudelka-Hep, in 'Chemical Sensors IV' Ed. M. Aizawa, M.A. Butler, P. Vanysek, N. Yamazoe, 196th Meeting of The Electrochemical Society, Inc., Honolulu, Hawaii, 1999, 99.

[9] M.W. Boden, A.D.C. Chan, S.M. Degnan, S.B. Ruiz, US pat. US5,637,642, 1997

[10] P.D. van der Wal, French pat. FR2792226, 2001.

[11] P. Reichmuth, P.D. van der Wal, M. Puntener, A. Schoning-Hammer, W.E. Morf, N.F. de Rooij, E. Pretsch, Anal. Chim. Acta 2002, 464, 79 . 